\title{
Phytophthora ramorum Does Not Cause Physiologically Significant Systemic Injury to California Bay Laurel, Its Primary Reservoir Host
}

\author{
M. V. DiLeo, R. M. Bostock, and D. M. Rizzo
}

University of California, Department of Plant Pathology, One Shields Ave., Davis 95616.

Current address of M. V. DiLeo: Boyce Thompson Institute for Plant Research, Tower Road, Ithaca, NY 14853-1801.

Accepted for publication 23 July 2009.

\section{ABSTRACT}

DiLeo, M. V., Bostock, R. M., and Rizzo, D. M. 2009. Phytophthora ramorum does not cause physiologically significant systemic injury to California bay laurel, its primary reservoir host. Phytopathology 99:13071311.

California bay laurel trees (Umbellularia californica) play a crucial role in the reproduction and survival of Phytophthora ramorum in coastal California forests by supporting sporulation during the rainy season and by providing a means for the pathogen to survive the dry, Mediterranean summer. While bay laurel is thus critical to the epidemiology of sudden oak death and other $P$. ramorum diseases in California, the relatively minor symptoms observed on this reservoir host suggest that it may not sustain ecologically significant injury itself. The long-term role that $P$. ramorum will play in California forests will depend in part on the extent to which this pathogen decreases the ecological fitness of bay laurel. Despite the importance of this question, no study has yet investigated in detail the physiological impact that ramorum blight imposes on bay laurel. This experimental study quantifies the impact that $P$. ramorum has on artificially inoculated bay laurel seedlings with measurements that integrate the full injury that infection with an oomycete may cause: photosynthetic efficiency, total photosynthetic area, and growth. Leaf area and leaf mass were not impacted significantly by infection of $P$. ramorum. Photosynthetic efficiency was mildly depressed in symptomatic, but not asymptomatic leaves, despite unnaturally high levels of necrosis that were imposed on the seedlings. These results demonstrate that bay laurel trees suffer only minor injury from ramorum blight beyond visible necrotic symptoms. Consequently, it is highly likely that bay laurel will continue to be widely available as a host for $P$. ramorum in California forests, which has long-term implications for the composition of these forests.

Additional keyword: ecophysiology.

Phytophthora species interfere with normal plant physiology not only by inducing necrosis but also by physically blocking intercellular spaces and vascular tissue and by secreting toxins $(17,23)$. Pathogen impacts on photosynthesis, respiration and water usage may occur both locally and systemically, with significant investments of energy from the plant for cellular reprogramming and altered metabolism in otherwise asymptomatic tissues $(11,24)$. Specifically, Manter et al. (20) demonstrated that the application of purified $P$. ramorum elicitins could significantly decrease the photosynthetic efficiency of uninfected bay laurel shoots in the absence of necrosis $(20,21)$. Therefore, the physiological impact of $P$. ramorum infection on bay laurel cannot be estimated solely by the extent of visible necrosis.

Although it is often assumed that $P$. ramorum does not exert an ecologically relevant impact on the fitness of native bay laurel trees, no experiments have investigated this quantitatively (5). Here, the impacts of $P$. ramorum infection on photosynthetic efficiency, total photosynthetic area, and growth of bay laurel seedlings were examined. In order to conservatively assess the resistance of bay laurel to $P$. ramorum, seedlings were exposed to levels of inoculum higher than occur in natural ecosystems under environmental conditions similar to those that typically occur during early spring epidemics. The long-term dynamics of $P$. ramorum diseases in California forests partially depend on the extent to which this pathogen negatively impacts the fitness of bay laurel. Accordingly, our results may help predict future ecological impacts and epidemiological behavior of $P$. ramorum in native California forests. As host dieback could presumably result in local extirpation of this pathogen, the long-term dynamics of these diseases can only be predicted with knowledge of the ecological impacts of P. ramorum on bay laurel. 


\section{MATERIALS AND METHODS}

Plant material. Nursery-grown bay laurel seedlings, approximately $40 \mathrm{~cm}$ tall, were purchased and maintained in a climatecontrolled greenhouse prior to experimentation. Seedlings were moved to a growth chamber with a $12 \mathrm{~h}$ photoperiod with $20^{\circ} \mathrm{C}$ days, $15^{\circ} \mathrm{C}$ nights, and $80 \%$ relative humidity 2 weeks prior to each of two trials. Isolated necrotic lesions and spots were present on most plants prior to experimentation. $P$. ramorum could not be isolated from any of these lesions, which appeared to be abiotic in etiology. Seedlings in both trials continued to grow actively in both the greenhouse and growth chamber.

Inoculation treatments. Three treatments were imposed: mock inoculation, single inoculation, and double inoculation. An inoculation cocktail was created by combining zoospores from three isolates of $P$. ramorum that were obtained from symptomatic bay laurel leaf lesions found in naturally infested forests in Sonoma County, CA, during the summer of 2005. Sporangia were produced from 3-day-old V8 agar cultures by transferring $3 \mathrm{~mm}$ plugs from the margin to sterile deionized water for 2 days. Zoospores were released by exposing the agar plugs to $4{ }^{\circ} \mathrm{C}$ for $20 \mathrm{~min}$ and adjusted to a final concentration of $2 \times 10^{5}$ zoospores per ml. Viability of all spore suspensions was confirmed with the observation of swimming zoospores.

Inoculated seedlings were sprayed with this zoospore suspension until runoff (approximately $10 \mathrm{ml}$ per plant) and enclosed in individual black plastic bags. Control seedlings were sprayed with deionized water. Each bag was opened daily and sprayed with deionized water for the subsequent 11 days in order to facilitate primary and secondary infection. Double inoculation seedlings were reinoculated between the second and third photosynthesis reading as described while control plants and single inoculated plants were sprayed with deionized water. Following each inoculation treatment, seedlings were removed from their bags and sprayed semiweekly with deionized water during the course of the experiment to produce an environment conducive to ramorum blight. Inoculation treatments were each applied to 15 seedlings in a completely randomized and balanced design in each of two trials.

Biomass analysis. At the end of each experiment, all seedlings were stripped of their leaves. Total leaf fresh weight was recorded for each seedling as leaf senescence is often marked by declines in leaf mass (28). Leaves were scanned into a digital image and measured with the image analysis program, APS Assess, to quantify total leaf area and total necrotic area. Percent necrotic area was calculated for each plant.

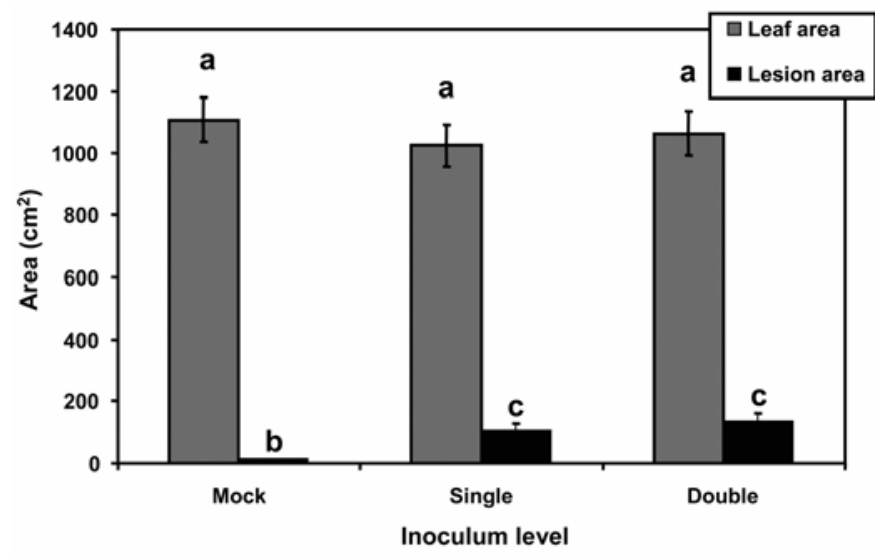

Fig. 1. Average total leaf and lesion area of bay laurel seedlings exposed to mock, single, or double inoculation of Phytophthora ramorum. Average total percent lesion area per seedling exposed to mock, single, and double inoculations was $1.2,12$, and $15 \%$, respectively. Letters indicate significant differences (mean $\pm \mathrm{SE}, n=30$ ). Trials were combined for analysis.
Photosynthetic efficiency. Maximum quantum yield (light harvesting efficiency, $\mathrm{F}_{\mathrm{v}} / \mathrm{F}_{\mathrm{m}}$ ) of open photosystem II centers was nondestructively measured with a LI-6400 fluorometer (LI-COR, Lincoln, NE) for two dark-adapted, asymptomatic leaves on each plant at the beginning of the experiment to confirm that equivalent leaves exhibited nearly identical values. A second reading was taken 2 weeks following the initial exposure to zoospores with a third reading taken 2 weeks following the second exposure to zoospores, over a total experimental period of 5 weeks. At each time point following inoculation, photosynthetic efficiency was measured on both asymptomatic regions of symptomatic leaves and on completely asymptomatic leaves. This experimental design allowed us to compare the impacts of $P$. ramorum infection both locally, on infected leaves, and systemically, on leaves distal from infection sites. All measurements were obtained from mature, fully expanded leaves, the developmental stage that most commonly displays ramorum blight symptoms in natural infections. As bay laurel is an evergreen, mature leaves that are less than 1 year old are indistinguishable from leaves that are several years old. Previous studies, however, have found that leaf properties vary little over this time period (12). Asymptomatic readings were taken on a single, healthy leaf lacking symptoms of abiotic or biotic stress on each seedling at each time point. For symptomatic readings, the single leaf with the greatest necrotic area on each plant, at each time point was chosen for measurement.

Statistical analysis. Leaf area, lesion area, and percent lesion area were analyzed with analysis of variance (ANOVA), and photosynthetic efficiency was analyzed with repeated measures MANOVA in JMP IN 5.1 for Windows (SAS Institute Inc., Cary, NC). Linear regression was performed on lesion area versus photosynthetic efficiency using Microsoft Office Excel 2003. Data from both trials were combined for analysis as blocks as they exhibited similar variances and lacked major trial by treatment interactions. All figures display the combined average of both trials.

\section{RESULTS}

Ramorum blight symptoms. All inoculated bay laurel seedlings developed characteristic symptoms of $P$. ramorum infection following exposure to zoospores, including limited leaf tip necrosis, small necrotic leaf spots, and dieback of immature leaves and shoot tips. Chlorosis, which is sometimes observed on naturally infected bay laurel, occupied an extremely small proportion of symptomatic leaf area, but was included in quantification of necrosis. Necrotic lesions developed within a few days of the first inoculation and did not appear to expand significantly during the ensuing month. Moreover, secondary infection after the primary inoculation did not appear to occur. Minor stem cankers were observed on one third of inoculated seedlings and one control seedling towards the end of the second trial, at which point they appeared to be expanding. $P$. ramorum was isolated from some cankers on inoculated seedlings. Fungal stromata were visible on all cankers, including one that was identified as a Sphaerosporium anamorph (2). P. ramorum was not isolated from any mock-inoculated control seedlings. Leaf abscission was not quantified as only a few leaves were lost among all seedlings.

Leaf area and mass. There were no significant differences in leaf mass $(P=0.4266)$ or leaf area $(P=0.7072)$ among treatments (Fig. 1). Leaves had significantly greater mass $(P=0.0002)$ and area $(P=0.0124)$ in the first trial.

Lesion area and percent lesion area. Inoculated seedlings had greater lesion area $(P<0.0001)$ and percent lesion area $(P<$ 0.0001) than mock-inoculated seedlings (Fig. 1). Single and double inoculated seedlings did not differ significantly in lesion area or percent lesion area. Average percent lesion area and standard deviation for mock-inoculated treatments was $1.2 \pm 1.8 \%$. Average percent lesion area and standard deviation for single and 
double inoculated seedlings were $12 \pm 15$ and $15 \pm 15 \%$, respectively. Lesion area and percent lesion area were significantly greater in the second trial (data not shown).

Photosynthetic efficiency. Photosynthetic efficiency $\left(F_{v} / F_{m}\right)$ of symptomatic leaves was less than that of asymptomatic leaves and decreased over time $(P<0.0001)$. Photosynthetic efficiency of symptomatic leaves did not differ between single and double inoculation treatments $(P=0.4873)$ but did change significantly over the course of the experiment $(P<0.0001)$ (Fig. 2). Symptomatic leaf average $\mathrm{F}_{\mathrm{v}} / \mathrm{F}_{\mathrm{m}}$ and standard deviation for single and double inoculated seedlings were $0.77 \pm 0.04$ and $0.75 \pm 0.13$, respectively. Photosynthetic efficiency of asymptomatic leaves did not differ significantly among inoculation treatments $(P=$ $0.4898)$ or over time $(P=0.0984)$ (Fig. 3). Photosynthetic efficiency was not well correlated with percent lesion area $\left(R^{2}=0.13\right)$ (Fig. 4). Asymptomatic leaf average $\mathrm{F}_{\mathrm{v}} / \mathrm{F}_{\mathrm{m}}$ and standard deviation for mock-inoculated seedlings was $0.79 \pm 0.04$. Asymptomatic leaf $F_{v} / F_{m}$ averages and standard deviations for single and double inoculated seedlings were $0.79 \pm 0.03$ and $0.79 \pm 0.09$, respectively. Symptomatic, but not asymptomatic, leaf photosynthetic efficiency varied significantly with trial $(P=0.0356)$ and had a significant interaction between trial and time $(P=0.0066)$.

\section{DISCUSSION}

Typical ramorum blight symptoms were expressed on all seedlings that were inoculated with $P$. ramorum. Approximately $14 \%$ of total leaf tissue became necrotic following inoculation, a number exceeding that which generally occurs in coastal California forests, where percent necrosis rarely exceeds $10 \%$ on symptomatic leaves, which often make up a very small proportion of total leaf area $(1,8)$. The high level of disease observed in this experiment can be attributed to inoculation conditions, and possibly phenology, as California $P$. ramorum populations are clonal and bay laurel populations lack geographic variation in susceptibility $(1,15,16)$. Additionally, a second inoculation did not significantly impact seedlings, suggesting that most of the susceptible tissue was colonized by the primary inoculation. Although it is possible that systemic acquired resistance operates in the interaction of $P$. ramorum and California bay laurel, it could not be detected under our experimental conditions (data not shown).

Seedlings used in the second trial exhibited lower leaf mass and area, and more severe disease symptoms and photosynthetic inhibition than seedlings used in the first trial. Seedlings used in the first trial were removed from the greenhouse in March while those used in the second trial were removed in June. Therefore,

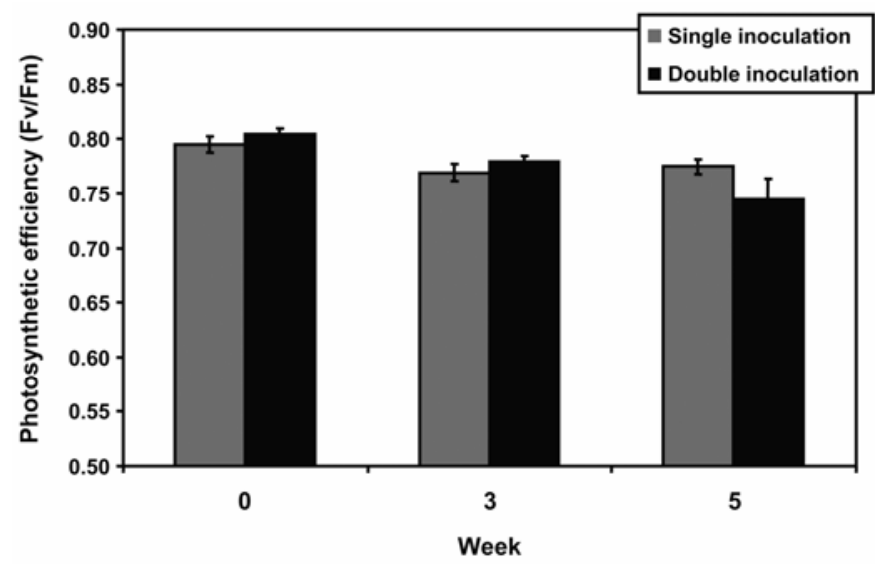

Fig. 2. Average photosynthetic efficiency of symptomatic leaves of bay laurel seedlings exposed to single or double inoculation of Phytophthora ramorum. Week 0 indicates pre-inoculation photosynthetic efficiency (mean $\pm \mathrm{SE}, n=$ 30). Trials were combined for analysis. although plants in both trials were exposed to mild, relatively low light greenhouse conditions for a few weeks in early spring, plants belonging to the second trial were additionally exposed to hotter, higher light greenhouse conditions during the early summer. Measured differences in bay physiology may have arisen due to stress or, alternately, to phenological differences, which are thought to influence natural epidemics (6). It is unknown how the susceptibility of bay laurel may be primed by the preinoculation environment, and consequently it is unknown which trial more closely approximated the physiological state of native bay laurel trees.

Cankers developed on some bay seedlings, a possible symptom of ramorum blight that has not been documented previously (5). As cankers were observed much more frequently on seedlings that were exposed to $P$. ramorum, it appears that this pathogen likely played a role in canker development. It is unknown whether $P$. ramorum initiated the cankers directly or predisposed the seedlings to infection by fungi. It is possible that $P$. ramorum is capable of creating multiple novel pathogen complexes with different pathogenic fungi (19). It is important to note that as these cankers occurred on green, pliable stems, any corresponding symptom on wild trees would likely appear on young shoots, not on fully lignified trunks or branches. Regardless, as ramorum blight-associated cankers have yet to be documented on wild bay laurel trees, it appears unlikely that this putative symptom is ecologically important in California forests.

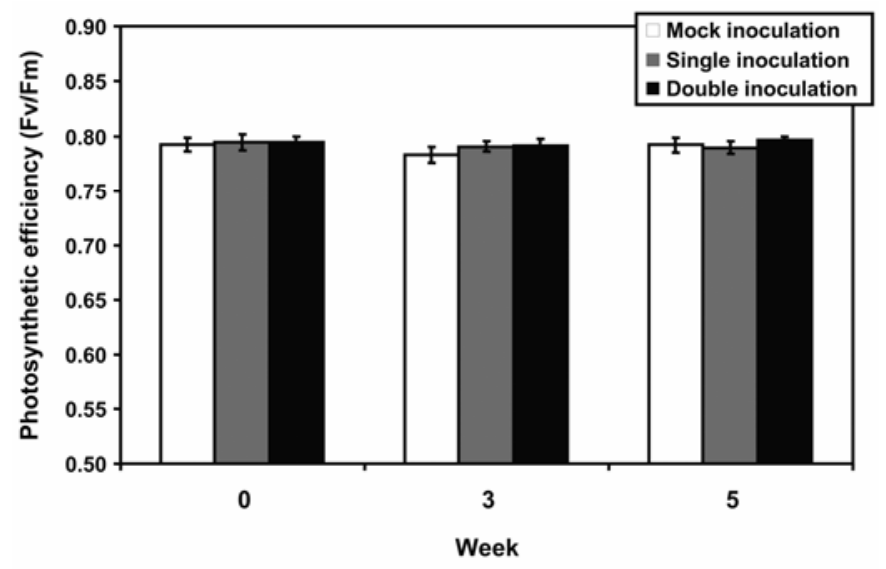

Fig. 3. Average photosynthetic efficiency of asymptomatic bay laurel leaves of seedlings exposed to mock, single or double inoculation of Phytophthora ramorum. Week 0 indicates pre-inoculation photosynthetic efficiency (mean \pm $\mathrm{SE}, n=30$ ). Trials were combined for analysis.

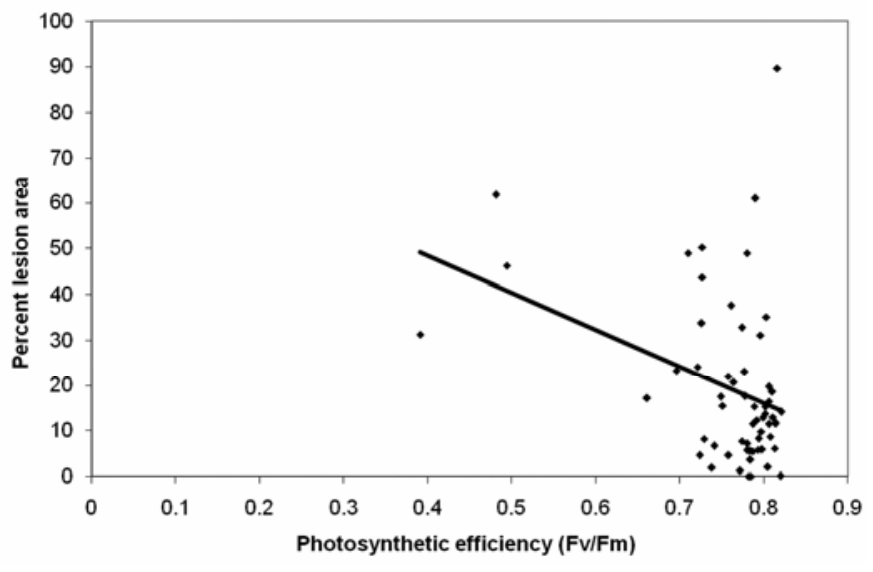

Fig. 4. Final photosynthetic efficiency of symptomatic bay laurel leaves versus percent necrotic area following inoculation with Phytophthora ramorum $\left(R^{2}=\right.$ $0.13, n=60)$. Trials were combined for analysis. 
Small, significant decreases in photosynthetic efficiency were detected in symptomatic leaves during this experiment, indicating stress from infection by $P$. ramorum. Measured values were found to be similar to those detected following the exposure of bay laurel shoots to $P$. ramorum elicitins (20). Accordingly, these decreases in photosynthetic efficiency may have been caused by pathogen produced effectors, such as elicitins. Although the in vivo spatiotemporal concentrations of $P$. ramorum-produced elicitins in bay laurel is not currently known, our results present strong evidence that any such toxins do not significantly impact bay laurel tissue more than a few centimeters from lesion margins. No significant differences were found among treatments for photosynthetic efficiency of asymptomatic leaves, suggesting an absence of systemic photosynthetic depression or compensation. That photosynthetic efficiency was not impacted even in heavily infected seedlings underlines the tolerance that bay laurel appears to exhibit to $P$. ramorum in addition to suggesting the absence of systemic pathogen-produced toxins. Additionally, although ramorum blight symptoms have been associated with abscission of bay laurel leaves in natural systems, only very limited leaf drop was observed in this study (6). As bay laurel is an evergreen that retains its leaves for up to 6 years, senescing leaves may simply be more susceptible to $P$. ramorum or, alternatively, senescence may be triggered in infected leaves (27).

Photosynthetic efficiency of asymptomatic regions of severely infected leaves at the final time point were, on average, $96 \%$ of the efficiency of asymptomatic leaves on either infected or uninfected bay laurel seedlings. It is important to note that small decreases in instantaneous photosynthetic efficiency may accumulate over time to produce large differences in carbon fixation, growth rate and resource allocation (10). However, in the absence of systemic photosynthetic depression, the subjection of a very small percentage of healthy tissue to inhibited photosynthesis and necrosis is ecologically insignificant relative to losses that bay laurel trees commonly encounter such as deer browse and abiotic hazards such as windthrow and wildfires $(13,26)$. Especially considering that inoculation conditions and symptom severity of this study were more extreme than occur in nature, and assuming no heretofore undetected impacts on flower production, fruit set, or seedling establishment, it appears unlikely that $P$. ramorum infestation of native forests would significantly decrease the fitness of naturally occurring bay laurel trees.

It is likely that bay laurel abundance in many of these natural forests will increase over time as competing oaks and tanoaks succumb to $P$. ramorum infection $(3,7,18)$. This ecological shift will be exacerbated if the maintenance or expansion of this reservoir of $P$. ramorum increases disease pressure on remaining oaks and tanoaks, or prevents the reestablishment of seedlings. Such changes in the ecological structure of these forests will further depend on the conduciveness of current and future forest microclimates, with the greatest ecological changes likely occurring at sites where bay laurel are most severely infected. Furthermore, although it is conceivable that some bay laurel trees subjected to the most severe infections could suffer significant injury due to accumulated photosynthetic inhibition, necrosis and, possibly, abscission, this would be mitigated by decreased competition from local oaks and tanoaks.

The future evolution of $P$. ramorum in California forests will depend at least in part on the selective constraints imposed by bay laurel, the epidemiologically dominant host. Accordingly, mortality associated with infection of tanoak and especially the epidemiological "dead-end" host, oak, may continue as long as bay laurel provides ubiquitous susceptible tissue and opportunities for transmission (29). Consequently, as long as susceptible host tissue remains abundant in these ecosystems, remaining tanoak and oaks may potentially be unable to escape infection by surviving at densities below what is required to support epidemics.

\section{ACKNOWLEDGMENTS}

We thank A. J. Bloom and L. Randall for the use of and assistance with the fluorometer, K. Aram for supplying P. ramorum cultures, and L. Yakabe for assistance with statistical analyses. This research was supported by the USDA Forest Service Pacific Southwest Research Station, the Gordon and Betty Moore Foundation, and the Department of Homeland Security.

\section{LITERATURE CITED}

1. Anacker, B. L., Rank, N. E., Huberli, D., Garbelotto, M., Gordon, S., Harnik, T., Whitkus, R., and Meentemeyer, R. 2008. Susceptibility to Phytophthora ramorum in a key infectious host: Landscape variation in host genotype, host phenotype, and environmental factors. New Phytol. 177:756-766.

2. Barnett, H. L., and Hunter, B. B. 1972. Illustrated Genera of the Imperfect Fungi. Burgess Publishing Company, Minneapolis, MN.

3. Brown, L. B., and Allen-Diaz, B. 2009. Forest stand dynamics and sudden oak death: Mortality in mixed-evergreen forests dominated by coast live oak. For. Ecol. Manage. 257:1271-1280.

4. Davidson, J. M., Patterson, H. A., and Rizzo, D. M. 2008. Sources of inoculum for Phytophthora ramorum in a redwood forest. Phytopathology 98:860-866.

5. Davidson, J. M., Werres, S., Garbelotto, M., Hansen, E. M., and Rizzo, D. M. 2003. Sudden oak death and associated diseases caused by Phytophthora ramorum. Plant Health Progress doi:10.1094/PHP-2003-0707-01-DG.

6. Davidson, J. M., Wickland, A. C., Patterson, H. A., Falk, K. R., and Rizzo, D. M. 2005. Transmission of Phytophthora ramorum in mixedevergreen forest in California. Phytopathology 95:587-596.

7. De Chant, T., Kelly, M., and Allen-Diaz, B. 2006. Gap dynamics in oak woodlands across a gradient of disturbance. Proceedings of the Sixth Symposium on Oak Woodlands: Today's challenges, tomorrow's opportunities. Pacific Southwest Research Station, Forest Service, USDA, Albany, CA.

8. DiLeo, M. V. 2008. Ecophysiological factors mitigating in planta survival of Phytophthora ramorum in California bay laurel. Ph.D. thesis. University of California, Davis.

9. Fichtner, E. J., Lynch, S. C., and Rizzo, D. M. 2007. Detection, distribution, sporulation, and survival of Phytophthora ramorum in a California redwood-tanoak. Forest Soil 97:1366-1375.

10. Fichtner, K., Koch, G. W., and Mooney, H. A. 1994. Photosynthesis, storage, and allocation. In: Ecophysiology of Photosynthesis. E. Schulze and M. M. Caldwell, eds. Springer-Verlag, Berlin

11. Goodman, R. N., Kiraly, Z., and Wood, K. R. 1986. The Biochemistry and Physiology of Plant Disease. University of Missouri Press, Columbia.

12. Goralka, R. J. L., Schumaker, M. A., and Langenheim, J. H. 1996. Variation in chemical and physical properties during leaf development in California bay tree (Umbellularia californica): Predictions regarding palatability for deer. Biochem. Syst. Ecol. 24:93-103.

13. Hepting, G. H. 1971. Diseases of Forest and Shade Trees of the United States. U.S. Department of Agriculture Forest Service, Washington, D.C.

14. Hickman, J. C., ed. 1993. The Jepson Manual: Higher Plants of California. University of California Press, Berkeley.

15. Ivors, K., Garbelotto, M., Vries, I. D. E., Ruyter-Spira, C., Hekkert, B. T., Rosenzweig, N., and Bonants, P. 2006. Microsatellite markers identify three lineages of Phytophthora ramorum in US nurseries, yet single lineages in US forest and European nursery populations. Mol. Ecol. 15:1493-1505.

16. Ivors, K. L., Hayden, K. J., Bonants, P. J. M., Rizzo, D. M., and Garbelotto, M. 2004. AFLP and phylogenetic analyses of North American and European populations of Phytophthora ramorum. Mycol. Res. 108:378-392.

17. Kamoun, S. 2006. A catalogue of the effector secretome of plant pathogenic oomycetes. Annu. Rev. Phytopathol. 44:41-60.

18. Maloney, P. E., Lynch, S. C., Kane, S. F., Jensen, C. E., and Rizzo, D. M. 2005. Establishment of an emerging generalist pathogen in redwood forest communities. J. Ecol. 93:899-905.

19. Maloney, P. E., Lynch, S. C., Kane, S. R., and Rizzo, D. M. 2004. Disease progression of Phytophthora ramorum and Botryosphaeria dothidea on pacific madrone. Plant Dis. 88:852-857.

20. Manter, D. K., Kelsey, R. G., and Karchesy, J. J. 2007. Photosynthetic declines in Phytophthora ramorum-infected plants develop prior to water stress and in response to exogenous application of elicitins. Phytopathology 97:850-856.

21. Maxwell, K., and Johnson, G. N. 2000. Chlorophyll fluorescence-A practical guide. J. Exp. Bot. 51:659-668.

22. Parke, J. L., and Lucas, S. 2008. Sudden oak death and ramorum blight. Online. The Plant Health Instructor doi:10.1094/PHI-I-2008-0227-01. 
23. Parke, J. L., Oh, E., Voelker, S., Hansen, E. M., Buckles, G., and Lachenbruch, B. 2007. Phytophthora ramorum colonizes tanoak xylem and is associated with reduced stem water transport. Phytopathology 97:1558-1567.

24. Prats, E., Gay, A. P., Mur, L. A. J., Thomas, B. J., and Carver, T. L. W. 2006. Stomatal lock-open, a consequence of epidermal cell death, follows transient suppression of stomatal opening in barley attacked by Blumeria graminis. J. Exp. Bot. 57:2211-2226.

25. Rizzo, D. M., Garbelotto, M., Davidson, J. M., Slaughter, G. W., and Koike, S. T. 2002. Phytophthora ramorum as the cause of extensive mortality of Quercus spp. and Lithocarpus densiflorus in California. Plant
Dis. $86: 205-214$.

26. Sampson, A. W., and Jespersen, B. S. 1963. California range brushlands and browse plants. University of California Extension Service, Berkeley.

27. Unsicker, J. E. 1974. Synecology of the California bay tree, Umbellularia californica (H. \& A.) Nutt. in the Santa Cruz Mountains. Thesis. University of California, Santa Cruz.

28. van Heerwaarden, L. M., Toet, S., and Aerts, R. 2003. Current measures of nutrient resorption efficiency lead to a substantial underestimation of real resorption efficiency: Facts and solutions. Oikos 101:664-669.

29. Woolhouse, M. E. J., Taylor, L. H., and Haydon, D. T. 2001. Population biology of multihost pathogens. Science 292:1109-1112. 